\title{
Modelling the Disk (three-phase) Interstellar Medium
}

\author{
Gerhard Hensler
}

Institute of Astronomy, University of Vienna, Türkenschanzstr. 17, 1180 Vienna, Austria email: hensler@astro.univie.ac.at

\begin{abstract}
The evolution of galactic disks from their early stages is dominated by gas dynamical effects like gas infall, galactic fountains, and galactic outflows, and more. The influence of these processes is only understandable in the framework of diverse gas phases differing in their thermal energies, dynamics, and element abundances. To trace the temporal and chemical evolution of galactic disks, it is therefore essential to model the interstellar gasdynamics combined with stellar dynamics, the interactions between gas phases, and star-gas mass and energy exchanges as detailed as possible. This article reviews the potential of state-of-the-art numerical schemes like Smooth-Particle and grid-based hydrodynamics and their ingredients, such as star-formation criteria and feedback, energy deposit and metal enrichment by stars, and the influence of gasphase interactions on the galactic gas dynamics and chemistry.
\end{abstract}

Keywords. ISM: kinematics and dynamics, ISM: structure, Galaxy: disk, Galaxy: evolution, galaxies: evolution, galaxies: ISM

\section{Introduction}

Galactic disks must have formed by dissipation and cooling of the galactic gas that remained after the first star-formation (SF) episode in the spheriodal component of galaxies and was mixed with the returned and metal-enriched stellar matter. Cooling enforced the further collapse, and angular momentum kept the radially extended disk rotationally supported. Since then the disk must have been continuously fed and refreshed by infalling intergalactic gas, but also depleted by galactic outflows. As known from our Milky Way since the 70's, but now with the availability of new spectral ranges (from the FIR/submm to X-rays) and also perceived in other disk galaxies, the Interstellar Medium (ISM) consists of a multi-phase gas (Ferrière 2001), conditioning SF in the cool molecular components and driving the ISM dynamically, mainly through its very hot component.

Besides external effects, structure and evolution of galaxies are primarily determined by the energy budget of its ISM. Reasonably, also the ISM structure and its radiative and kinetic energy contents are both determined by the energy deposit of different sources on the one hand, and by the energy loss through radiative cooling on the other.

The state of the ISM is thus not an isolated and meaningless question. Since stars are born from the cool gas phase, but energize the ISM by means of their energy and mass release, the treatment of the ISM must ultimately include also the stellar component with its distribution in space, age, and metallicity. Disk formation and evolution thus depend intimately on the SF and the energy content, so that numerical simulations must account for these facts properly. These are, however, the most challenging tasks for theoretical and numerical astrophysics of the ISM and are fundamental for galaxy evolution.

Why is this so? On the one hand, the SF rate in disks obeys the well-known SchmidtKennicutt law (Kennicutt 1998) that relates the $\mathrm{H} \alpha$ surface brightness of disks with the gaseous surface density $\Sigma_{g}$; on the other hand, we know from theory and empirical 
results how much energy is released by stars during their different evolutionary stages. So, where is the problem? Is it not sufficient to relate the SF rate empirically to gas mass from studies of galaxies in the present local universe? Why should a detailed treatment of the ISM and its particular and temporal composition be necessary, not just a large-scale average of the state of the ISM?

In the following, let us reflect and comment on the present state of the numerical treatment of the multi-phase ISM with its dynamics, its SF and energy feedback, its necessary small-scale physical interaction processes, and the application to galaxy evolutionary models. After having explained the most important effects of the ISM on galaxy evolution and their empirical issues from observations, we will consider representative numerical developments and discuss their drawbacks and advantages without claiming completeness. Finally, we discuss the most advanced numerical efforts relative to the requirements for a fully realistic approach. Nevertheless, let me concede already in advance that the influence of magnetic fields and of Cosmic Rays are not yet included, and not even explored to first order in simulations.

\section{Structural and evolutionary effects on the Interstellar Medium}

\subsection{Star formation and the Schmidt-Kennnicutt Law}

Already in 1959 Schmidt argued that the SF rate per unit area relates to surface gas density $\Sigma_{\mathrm{g}}$ by a power law with exponent $n$. The Kennicutt (1998) relation

$$
\Sigma_{\mathrm{H} \alpha}\left[M_{\odot} /\left(y r \cdot p c^{2}\right)\right] \propto \Sigma_{\mathrm{g}}\left[M_{\odot} / p c^{2}\right]^{1.4 \pm 0.15}
$$

established that in equilibrium states the amount of gas determines the SF rate. Although this connection looked already pretty tight and holds over more than 4 orders of magnitude in $\Sigma_{\mathrm{g}}$ with a lower cut-off at almost $10 M_{\odot} / p c^{2}$, recent advances in observation and theoretical interpretation are more confusing. Heyer et al. (2004) showed that this exponent is only valid for the molecular gas and that the correlation is tighter, while for pure Hi the slope is steeper. Gao \& Solomon (2004) derived the dependence of the SF rate on the dense molecular cloud cores traced by the HCN molecule.

Such a Kennicutt relation can be theoretically simply supported as

$$
\Sigma_{\mathrm{SF}}=\frac{\Sigma_{\mathrm{g}}}{\tau_{\mathrm{SF}}} \propto \frac{\Sigma_{\mathrm{g}}}{\rho_{\mathrm{g}}^{-0.5}} \propto \Sigma_{\mathrm{g}}^{1.5}
$$

if the scaleheight $\mathrm{H}$ is independent of $\Sigma_{\mathrm{g}}$, i.e. the gas temperature is constant, so that $\Sigma_{\mathrm{g}} \propto \rho_{g, 0} \propto \rho_{\mathrm{g}}$. This can only be understood if heating and cooling both are independent of volume gas density $\rho_{\mathrm{g}}$ or have the same dependence on it. Köppen, Theis, \& Hensler (1995), however, demonstrates that the SF rate depends on $\rho_{\mathrm{g}}^{2}$, if the stellar heating is compensated by collisionally-excited cooling radiation (according to Böhringer \& Hensler 1989). The coefficient of this relation determines the SF timescale $\tau_{\mathrm{SF}}$.

But how can this be described? Elmegreen (2002) argued that the SF is determined by the free-fall time $\tau_{\mathrm{ff}}$. This, however, raises a conflict between the ISM conditions and observed SF rates in the sense that $\tau_{\mathrm{ff}}$ for a typical density of $100 \mathrm{~cm}^{-3}$ amounts to $10^{14}$ sec, i.e. $3 \cdot 10^{6}$ yrs. For a galactic molecular cloud mass of $10^{9} \ldots 10^{10} M_{\odot}$ the SF rate should amount to $10^{2} \ldots 10^{3} M_{\odot} / \mathrm{yr}$ - orders of magnitudes higher than observed! This means that the SF timescale must be reduced with respect to the collapse or dynamical timescale by introducing an efficiency $\epsilon_{\mathrm{SF}}$; its definition could be : $\tau_{\mathrm{SF}}=\epsilon_{\mathrm{SF}}^{-1} \cdot \tau_{\mathrm{ff}}$. Nevertheless, Li, MacLow, \& Klessen (2005), from numerical models of gravitationally unstable clouds, also obtained a Kennicutt law slightly steeper than $n=1.4$. 
Since this Schmidt-Kennicutt law is derived for gravitationally settled gas disks in rotational equilibrium, it should also reflect an equilibrium state of a self-gravitating vertically stratified ISM balanced by heating and cooling. It is therefore not surprising that the exponent is larger for starburst galaxies, i.e. if the SF is e.g. triggered by dynamical processes, than in a quiescent mode, so that the SF efficiency is increased. This also explains why high-z galaxies reveal a steeper relation with an exponent $n=1.7 \pm 0.05$ (Bournaud, Elemegreen, \& Elmegreen (2007)), i.e. at stages when the gaseous disks form preferably by gas infall.

One would reasonably expect that the Kennicutt relation can only be reproduced in self-regulated disk models, i.e. in those where energy feedback by stars determines the energy budget of the ISM. Kravtsov (2003) identified disk galaxies in his cosmological simulations and analyzed them at different high redshifts. His model parameters covered the large range observed in $\Sigma_{\mathrm{g}}$, and the model values scatter around the Kennicutt slope of $\Sigma_{\mathrm{SF}}$ with $\Sigma_{\mathrm{g}}$. However, surprisingly, models without energetic feedback by supernovae (SNe) did not differ from those with feedback. Similar conclusions can be drawn from the simulations by Schaye \& Dalla Vecchia (2008), who neglect stellar feedback processes while varying parameters like SF efficiency, critical SF mass, etc.

It is therefore still a matter of debate whether the Kennicutt-like dependence of the surface SF rate is really a result of self-regulated SF in gas disks, or if any dependence of self-regulation processes on the physical gas state cancel out, so that the relation is really a global one.

For modelling the formation and evolution of galactic disks, however, the column density is meaningless in systems still developing dynamically. As long as the disk has not achieved its equilibrium state for the gas it is therefore necessary, and from a physical standpoint reasonable, to link the SF rate to the local state within a volume. (By the way, the same should hold for the modelling of gaseous dwarf galaxies (DGs) which are always in the state of re-arranging their gas structure because of the low gravitational potential.) This, however, means that the SF rate in a gas volume, $\partial \rho_{\mathrm{SF}} / \partial t$, should be connected to the gas volume density $\rho_{\mathrm{g}}$ by a power-law of exponent $m$. But what is the value of $m$ ? Rana \& Wilkinson (1986) found a wide range of $m=1 \ldots 4$. Similar to the above derivation of the Kennicutt exponent, one can formulate

$$
\frac{\partial \rho_{\mathrm{SF}}}{\partial t}=\frac{\rho_{\mathrm{g}}}{\tau_{\mathrm{SF}}} \propto \frac{\rho_{\mathrm{g}}}{\rho_{\mathrm{g}}^{-0.5}} \propto \rho_{\mathrm{g}}^{1.5}
$$

The proportionality again includes the SF efficiency. Such a 1.5 power-law was already applied for this reason in the first gas dynamical simulations of galaxy evolution by Larson (1969 and in the 70's) and Burkert \& Hensler (1987, 1988). Under the assumption of triggered SF due to cloud-cloud collisions, a square dependence on $\rho_{\mathrm{g}}$ should apply. In a simple picture, moreover, the cloud collision rate depends not only on the cloud number density but also on the clouds' velocity dispersion, and can thereby directly affect the $\mathrm{SF}$ rate as a positive energetic feedback. Since dissipative collisions, however, lead to a heating of the cloudy material, and if the SF rate depends on the temperature of the cloudy ISM component, also a negative feedback is implied. Therefore, a competition between collisional heating and gas cooling will determine the sign of the SF feedback on energy and thus depends on the state and composition (metallicity, dust content, molecules, etc.) of the ISM.

Concerning the balance between direct stellar energy release to the ISM and collisionally-excited radiative cooling, Köppen, Theis, \& Hensler (1995) found that for the starforming site itself, self-regulation sets the dependence of the SF rate on $\rho_{\mathrm{g}}^{2}$. In addition, the production of a SN-heated hot gas phase and its interaction with the cool gas by heat 
conduction leads to a limit-cycle SF rate around a characteristic temperature due to the competition between evaporation and condensation (Köppen, Theis \& Hensler 1998).

Consequently, the energy release and the efficiency of its transfer into dynamics and thermalisation are of evident importance. If their description and parametrization as well as a Schmidt-dependent SF rate, respectively, are treated properly in numerical simulations, i.e. close to real conditions, a Kennicutt dependence for a gaseous galactic disk should inherently be reproduced.

\subsection{Stellar energy release and its transfer efficiency}

Because of their high power of released energy in various forms, massive stars are usually included as the only heating sources for the ISM. Moreover, a large fraction of simulations focuses the energy deposit on SN typeII explosions alone. It must be discussed, however, how effectively this energy is transferred into the ISM as turbulent and hence thermal energy, and an even more sophisticated question must be asked on the influence, transport and dissipation by Cosmic Rays and magnetic fields.

The same must be considered for the energy release of massive stars as radiation-driven and wind-blown Hurregions. Analytical estimates for purely radiative Huregions yielded an energy transfer efficiency $\epsilon$ of the order of a few percent (Lasker 1967). Although the additional stellar wind power $L_{w}$ can be easily evaluated from models and observations, the fraction that is transferred into thermal energy $\left(\epsilon_{t h}\right)$ or into turbulent energy is not obvious from first principles. Transfer efficiencies for both radiative and kinetic energies remain much lower than analytically derived from detailed numerical simulations (more than one order of magnitude) and amount to only a few per mil (Hensler 2007 and references therein; Hensler et al. 2009, in preparation). Surprisingly, there is almost no dependence on the stellar mass, (Hensler 2007) as expected because the energy impact by Lyman continuum radiation and wind luminosity both increase non-linearly with stellar mass. Conversely, since the gas compression is stronger by a more energetic wind, the energy loss by radiation is plausibly also more efficient.

Another fact is the energization of the ISM by SNe. Although it is generally assumed that the explosive energy lies around $10^{51}$ ergs with an uncertainty (or intrinsic scatter) of probably one order of magnitude, SNeII do not explode as isolated and single events in the ISM. Almost without exception, massive stars remain close to the SF site and thus explode within their stellar associations. Massive stars contribute significantly to the formation of structures, like e.g. cavities, holes, and chimneys in the HI gas and superbubbles of hot gas (Recchi \& Hensler 2006a). On large scales the energy release by massive stars triggers circulation of matter via galactic outflows from a gaseous disk and galactic winds. By this, also the chemical evolution is affected through the loss of metal-enriched gas from a galaxy (see e.g. Recchi \& Hensler 2006b).

SN explosions as an immediate consequence of SF stir up the ISM by the expansion of hot bubbles, deposit turbulent energy into the ISM and, thereby, regulate the SF again (Hensler \& Rieschick 2002). This negative energy feedback is enhanced at low gravitation, because the SN energy easily exceeds the galactic binding energy and drives a galactic wind. This low gravitation also exists in vertical direction of a rotationally supported disk. Conversely, SN and stellar wind-driven bubbles sweep up surrounding gas and can thereby excite SF self-propagation as a positive feedback mechanism (e.g. Ehlerova et al. 1997, Fukuda \& Hanawa 2000).

Since the numerical treatment of the galactic chemo-dynamical evolution, even when it deals with two gas phases, cannot spatially resolve the ISM sufficiently, for a detailed consideration of small-scale processes the chemo-dynamical modeling has to apply parametrizations of plasma-physical processes. It should, however, be repeatedly 
emphasized that an appropriate chemo-dynamical description cannot vary those parameters arbitrarily, because they rely on results from theoretical, numerical, and/or empirical studies and are thereby strongly constrained. This has been described and applied to different levels of chemo-dynamical models (for an overview see Hensler 2003 and references therein, and more recently Hensler, Theis \& Gallagher 2004a; Harfst, Theis \& Hensler (2006)).

Investigations have been performed for $\epsilon$ of SNe (Thornton et al. 1998) and superbubbles (e.g. Strickland et al. 2004), but they are yet too simplistic for quantitative results, and the $10 \%$ efficiency derived by Thornton et al. is far too large. Although numerical experiments of superbubbles and galactic winds in galaxies are performed, yet they only demonstrate the destructive effect on the surrounding ISM, but lack self-consistency and complexity.

Simulations of the chemical evolution of starburst DGs by Recchi et al. (2006), aimed at reproducing the peculiar abundance patterns in these galaxies by different SF episodes, found that $\epsilon$ can vary widely. A superbubble which acts against the surrounding medium is cooling due to its pressure work and radiation, but compresses the swept-up shell material and imparts significant turbulent energy to the ISM. If a closely following SF episode (maybe another burst) pushes its explosive SNeII into the already formed chimney, the hot gas can easily escape without hindrance. Recchi \& Hensler 2006a found that, depending on the external Hi density, the chimneys do not close before a few hundred Myrs.

\subsection{Gas Infall}

Formation and evolution of galactic disks cannot be considered in isolation. As confirmed from observations, but also required in the $\Lambda$ CDM framework, galaxies grow continuously during their evolution by accreting surrounding gas. Particularly during the early formation phases it still remains unclear, however, how this process works. Cold and hot accretion are proposed (see e.g. Keres et al. 2005, Wise \& Abel 2007), both dependent on mass and providing different inherent timescales of the galaxy growth, but no clue yet exists which one dominates.

In the local Universe several disk galaxies are enveloped by huge Hi halos. Refined velocity-position maps reveal cold accretion in the form of Hi clouds with masses of about $10^{7} M_{\odot}$ (Fraternali et al. 2002; Fraternali, this volume, and e.g. Sancisi et al. 2008). Since the infall rate determined by the detectable clouds fails to support the SF rate in those galaxies by almost one order of magnitude, it must be concluded that most of the infall happens by less massive clouds, perhaps like those observed in our Milky Way.

Although the element abundance properties of most Blue Compact DGs (BCDs) favour a pure young stellar population of at most 1-2 Gyr, they mostly consist of an underlying old population. Furthermore, most of these objects are also embedded into Hi envelops from which at least NGC 1569 definitely suffers gas infall (Stil \& Isreal 2002, Mühle et al. 2005). This lead Köppen \& Hensler (2005) to exploit the influence of gas infall with metal-poor gas into an old galaxy with continuous SF on particular abundance patterns. Their results could match not only the observational regime of BCDs in the $[12+\log (\mathrm{O} / \mathrm{H})$ $-\log (\mathrm{N} / \mathrm{O})]$ space but also explain the shark-fin shape of the data distribution. Knauth et al. (2006) also found peculiar N/O abundance ratios in the galactic ISM, which could be explained by the infall scenario of Köppen \& Hensler (2005).

The accumulated explosion of massive stars lead to the formation of a superbubble which expands out of the gaseous disk, preferentially along the steepest density gradient. In low-density environments when the thermal energy does not exert too much $\mathrm{PdV}$ work or lose significant radiative energy, this expansion feeds the galactic halo with hot 
gas. On the other hand, Hi observations have long confirmed that high- und ultra-high velocity clouds of extragalactic origin fall towards the Galactic disk, demonstrating that the halo consists of a multi-phase ISM (Wolfire et al. 1995). Interstellar clouds with moving relative to the surrounding gas are deformed and stretched by the ram pressure. Heyer et al. (1996) showed the cometary deformation of clouds exposed to the outflow of hot gas in the SF region W4. Infalling clouds are not only influenced by a drag force of the hot halo gas because of its relative velocity and thereby decelerated (Benjamin \& Danly 1997), the thermal interaction between the gas phases through heat conduction also affects their survival (Vieser \& Hensler 2007). Due to their possible destruction and gas dispersion in hot environments, it is in general far from clear how many of the infalling high-velocity clouds really reach the gaseous disk, feed the SF, and excite turbulence, neither in the early galaxy growth phase nor during the present evolution.

\subsection{Galactic Winds}

Some disk galaxies, like e.g. NGC 253 and M82, not only supply hot halo gas but can also drive a galactic wind. This scenario can be observed in various types of galaxies and in different evolutionary stages. In the Galactic disk cavities and chimneys become visible in $\mathrm{HI}$, and in external galaxies Hi holes appear. In DGs, superbubbles originating from super star clusters (SSCs) are observable as closed $\mathrm{H} \alpha$ loops and in X-rays still confined to the galaxy, like in NGC 1705 (see e.g. Hensler et al. 1998). NGC 1569 is a most interesting object: not only does gas infall occur and probably triggers the present starburst (Mühle et al. 2005), but a strong galactic wind also exists. An analysis of the wind revealed a metal content only $\sim 1-2$ times solar, i.e. much lower than expected from the yields by massive stars (Martin, Kobulnicki \& Heckman 2002). Since this low value requires dilution with low-metallicity gas by a factor of about 10 , turbulent mixing alone in the shells of the expanding superbubble with the low-Z ISM in NGC 1569 seems insufficient. More plausibly, gas clouds falling in from the enveloping HI reservoir evaporate and thereby mass-load the galactic wind.

\subsection{Structural issues}

Summarizing the above-mentioned observational facts, one must be aware that:

- the multi-phase structure of the ISM exists in any galactic region;

- the mode of SF and its positive or negative energetic feedback depend on the smallscale physics of the ISM; and

- the reliability and significance of simple, single-phase hydrodynamical models, even with feedback, are highly questionable.

\section{Numerical Methods}

\subsection{Star-formation prescriptions}

There are vigorous efforts to model the ISM on small scales in star-forming clouds in order to achive a deeper insight into the mechanisms controlling SF (see e.g. Ostriker, Stone, \& Gammie 2001, MacLow \& Klessen 2004, Li, MacLow \& Klessen 2005, Krumholz \& McKee 2005). Numerical simulations on intermediate scales must already prescribe the SF by some reasonable recipe. Numerous papers (see e.g. Wada \& Norman 1999, de Avillez \& Breitschwerdt 2004, Slyz et al. 2005, Tasker \& Bryan 2008) have implemented SF criteria like e.g. threshold density $\rho_{\mathrm{SF}}$ with stars forming if $\rho_{\mathrm{g}} \geqslant \rho_{\mathrm{SF}}$, excess mass in a specified volume with respect to the Jeans mass, i.e. $\mathrm{M}_{\mathrm{g}} \geqslant M_{\mathrm{J}}$, convergence of gas flows $($ div $\cdot \underline{\mathbf{v}}<$ 0 ), cooling timescale $t_{\text {cool }} \leqslant t_{d y n}$, temperature limits $\mathrm{T}_{\mathrm{g}} \leqslant \mathrm{T}_{\text {lim }}$, Toomre's Q parameter, and/or temperature dependent SF rate. If at least some of these conditions are fulfilled, 
as a further step the gas mass which is converted into stars, i.e. the SF efficiency, has to be set. An empirical value of $\epsilon_{\mathrm{SF}}$ is fixed from observations, i.e. $\Delta m_{\mathrm{SF}}=\epsilon_{\mathrm{SF}} \cdot \rho_{\mathrm{g}} \cdot \Delta x^{3}$, where $\Delta x^{3}$ is the mesh volume in a grid code. If the numerical timestep $\Delta t$ is smaller than the dynamical timestep $\tau_{\mathrm{ff}}, \Delta m_{\mathrm{SF}}$ must be weighted by their ratio (Tasker \& Bryan 2008). Since $\epsilon_{\mathrm{SF}}$ must inherently depend on local conditions, such that it is high in bursting $\mathrm{SF}$ modes as required for the Globular Cluster formation, but low (a few percent) in the self-regulated SF mode, numerical simulations often try to derive a realistic SF efficiency by comparing models of widely different $\epsilon_{\mathrm{SF}}$ with observations (see e.g. Tasker \& Bryan 2008: $\epsilon_{\mathrm{SF}}=0.05$ and 0.5$)$.

While the Kennicutt law (Schaye \& Dalla Vecchia 2008) is directly applied to the SF rate in many numerical treatments as the simplest prescription, another approach is a formulation based on eq. (2.3), like

$$
\frac{\partial \rho_{\mathrm{SF}}}{\partial t}=\alpha G^{1 / 2} \rho_{\mathrm{g}}^{3 / 2}
$$

and to express $\alpha$ using the Kennicutt law again and applying an exponential disk with scaleheight $Z_{0}$ by

$$
\alpha=1.96 \cdot G^{-1 / 2} \frac{\Sigma_{\mathrm{SF}}}{\Sigma_{\mathrm{g}}^{3 / 2}} Z_{0}^{1 / 2}
$$

But, unreasonably, this is even applied to interacting galaxies, (Springel 2000) where both the application of the Kennicutt law and the usual definition of scale height is highly questionable, and in my opinion fails.

Theoretical studies by Elmegreen \& Efremov (1997) derived a dependence of $\epsilon_{\mathrm{SF}}$ on the external pressure, while Köppen, Theis, \& Hensler (1995) explored a temperature dependence of the $\mathrm{SF}$ rate.

\subsection{Smooth particle hydrodynamics}

Because of its simple numerical treatment and inherent 3D representation, Smooth Particle Hydrodynamics ( $\mathrm{SPH}$ ) is at present the most widely applied numerical strategy for simulations of cosmological structure formation, galaxy formation, galaxy collisions and mergers, and so forth. Because of their particle character, SPH simulations easily allow simultaneous treatment of the gas and the stellar component. The SPH subdivides the gaseous component in gas packages of sizes that represent the continuum character of diffuse gas, i.e. by large spherical extents of the SPH particles, expressed by a kernel function $W_{i j}$ so that the mass density at particle $\mathrm{i}$ is given through the neighbouring particles $\mathrm{j}$ with mass $m_{j}$ by

$$
\bar{\rho}_{i}=\sum_{j=1}^{N} m_{j} \cdot W_{i j} .
$$

Although SPH is a quite powerful formalism for 3D problems, is has several drawbacks: 1) On the one hand, representing gas phases which change their scalelengths by orders of magnitude within a short range, i.e. adjacent hot and cool particles, is impossible. Unfortunately, many authors are misled in such applications. 2) Ad hoc assumptions on $\mathrm{SF}$ and feedback are necessary. As an example: over which range, i.e. over how many adjacent particles, must the SNII energy feedback be taken into account? If the mass and spatial resolution of SPH particles is insufficient, the mean thermal energy of a gas particle is forced to not increase due to SNeII, but will remain at about $10^{4} \mathrm{~K}$ due to efficient cooling. The coarse treatment is therefore insensitive to self-regulation, and thereby cannot account for a hot gas phase. 3) Multi-fluid descriptions are almost 
impossible. If one decouples two kinds of particles totally, they can hardly experience the proximity of each other. Or one allows for different states but one kind of particle, so that each particle acts to its adjacent neighbour as a continuous fluid.

Because these weaknesses, which are mostly caused by the single gas-phase description and the lack of interaction processes, have been recognized by many modellers, mainly also in the field of cosmological simulations, numerous attempts to overcome them have been performed, developing various strategies for the treatment of a multi-phase ISM. Since GADGET is probably the most widely distributed and -applied publicly available SPH code (Springel 2000, Springel, Yoshida, \& White 2001) let us consider specifically its application to disk galaxies and its advancements.

Dalla Vecchia \& Schaye (2008) applied GADGET to study the impact of SN feedback on galactic disks in order to drive a galactic wind, and conversely the effect of the wind strength on the SF. The latter is described by the Kennicutt law combined with a density threshold. For massive galaxies with DM mass of $10^{12} M_{\odot}$, the mass resolution of gas particles extends down to $5 \cdot 10^{4} M_{\odot}$. The SNII energy is deposited as kinetic wind energy, and wind particles are stochastically selected among neighbouring particles. In some models these are hydrodynamically coupled to the normal gas particles. As expected, the models show that galactic winds are stronger for larger energy release and, on the other hand, reduce the SF rate, but not in a self-consistent manner, because neither cold-gas infall nor SF trigger is included. In addition, in models with a wind the SF rates decrease by about a factor of two already within 0.5 Gyrs, which is much too short for derived SF timescales of disk galaxies.

In another recent development, Scannapieco et al. (2006) use a very sophisticated differentiation between the SPH particles into cold and hot ones, but still treat them adequately, except that particles with different thermodynamic variables (cold vs. hot) do not feel each other. The recipe to allow for SNII energy to be distributed into the cold and hot particles, but not cooled away in the cold ones, is the possibility of a so-called promotion of cold particles according to the state of their hot neighbors. Scannapieco et al. (2006) claim that even mass-loaded galactic winds can develop in these models. Nevertheless, SF criteria and efficiency as well as the distribution of the SN energy are freely parametrized and adapted to galaxies in the local Universe observed in equlibrium states.

In order to describe the different ISM phases by at least two particles, also differently treated, Semelin \& Combes (2002) introduced four different particle species: warm gas as SPH particles, stars and DM as collisionless particles, and cold gas as sticky particles. Mass exchange between the cold and warm gas is limited by the transformation by heating from SN feedback vs. cooling. The cool particles are not intended to represent a diffuse gas distribution, but instead the clumpiness of the cloudy ISM component, and thus subject to individual cloud-cloud collisions with only partial inelasticity.

Further mass transfer between the components is allowed by SF and stellar mass loss. While the SF rate is described by a Schmidt law of the cool gas volume density with a power of 1 to 1.5, the mass fraction of formed stars is at first trapped within the cool gas particle. Not until a sufficient amount of stars exists in a specified number of neighbouring particles are these stellar masses subtracted from the gas particle and collected in a new, pure stellar particle. Semelin \& Combes (2002) applied an advanced procedure to keep the particle number almost constant. Further mass exchange is achieved by the stellar mass return according to the stellar evolution and death rate. The particles are numerically treated by the so-called TREE algorithm (Barnes \& Hut 1986).

Although this approach looks well justified and explored, the illustration of the best model over 2 Gyrs in Semelin \& Combes (2002) shows that it obviously suffers from an 
unrealistically high SF rate. The gas is consumed to $20 \%$ within this short time and the SF rate has dropped from almost $100 M_{\odot} /$ yr to less than 5 . The corresponding e-folding timescale of SF amounts to only less than 0.5 Gyrs, which stands in contradiction to the observationally derived value, e.g. in the Milky Way.

This model behaviour of a too intensive SF might have different reasons: no dynamical, energetic and matter interactions are implied into the numerical treatment. Dynamically, the drag exerted by hot gas flow against cool clouds is not sufficiently represented by only the thermal pressure gradient built up by the local SN energy release and driving the expansion of hot gas. From the energetic point of view, heat conduction transfers energy from the hot to the cool clouds and thereby regulates the SF (Köppen, Theis \& Hensler 1998). In addition, heat conduction also can lead to partial evaporation of clouds and thus reduce the cloud mass reservoir available for SF.

Surprisingly, even recently the formation of exponential disks has been modelled by Bournaud, Elemegreen, \& Elmegreen (2007) applying another speciality of algorithms, a particle-mesh + sticky-particle scheme. Although they dealt with the enormous number of one million particles for DM, gas and stars, their limitation to a single gas phase without any stellar feedback are much too severe to allow reliable results, in particular, with respect to the aimed issues of this exploitation. The author aimed at understanding the evolution of clump-clusters and chain galaxies by fragmentation of gaseous disks in gravitational unstable clumps. The reason for this neglect, that the feedback from SF "is not well known" and that the clump masses lie much above the influential range of SN energy, is misleading as far as it has not been tested and proven that the coupling of energetic processes between scales is really inefficient.

All the above mentioned additional processes, acting among the gas phases and, by this, coupling them even on different scales are additionally incorporated in the $\mathrm{SPH} /$ sticky particle treatment by Harfst, Theis \& Hensler (2006). Moreover, in their model the cloudy particles show an inherent mass distribution according to their stickiness, and SF can happen in a "normal" mode, but also be induced by cloud collisions. In addition to the temperature criterion by Köppen, Theis, \& Hensler (1995), the efficiency by Elmegreen \& Efremov (1997) controls the SF. As a result, Harfst, Theis \& Hensler (2006) demonstrated a stabilized rotating disk with moderate SF over 2 Gyrs, starting with a present-day realistic value of $3 M_{\odot} / \mathrm{yr}$ for a gaseous disk and dropping off only by a factor of 4 , which means a SF timescale of more than 1 Gyr. As argued by them, the reduction in the SF rate is caused by a lack of external gas that would be allowed to fall in, as it happens to the Milky Way and other disk galaxies, and maintain the SF. As a proxy, also the validity of the Kennicutt law could almost be reproduced, but with a slope of -1.5 .

The model treatment allows the mass release by massive and intermediate-mass stars, the latter as Planetary Nebulae (PNe), while the energy feedback is only attributed to SNeII by $20 \%$ of the normally used SNII energy of $10^{51}$ ergs. Until now, none of the last-mentioned numerical treatments includes type Ia SNe. Semelin \& Combes (2002) trace the metal enrichment from stellar ejecta as a simple generalized Z; Harfst, Theis \& Hensler (2006) do not yet, although their prescription would allow for the differential treatment of abundance yields.

This task has been performed until now only in a further advanced development by Berczik et al. (2003), Berczik et al. (2009), which is based on a single gas-phase SPH description by Berczik (1999). The treatment is almost the same as in Harfst, Theis \& Hensler (2006), except that here the cool gas phase is also treated by SPH particles. This strategy can, however, be justified by the still large minimum masses of the gas packages, $10^{5} M_{\odot}$, i.e. in the range of Giant Molecular Clouds (GMCs) instead of small SF clumps. 
Therefore, also the cool gas can be considered as continuous. The gas phases can behave dynamically independently and are only coupled by drag and mass loading by means of heat conduction. For the SF the Jeans mass criterion is used again

The most important advancement is the chemo-dynamical treatment, which includes elements released by different progenitor stars as PNe, SNeIa, SNeII, and stellar winds and enables one to trace the galactic evolutionary epochs.

\subsection{Grid codes}

The advantages of particle codes, due to the easy numerical handling and the simple inspection, is paid by the drawbacks of complication with the multi-phase treatment of the ISM, the poor spatial resolution, and with necessary physical processes acting and featuring the gas on various length scales. For these purposes, numerical codes based on spatial grids are at the moment more adaptable to higher resolution and more appropriate for the physics. Nevertheless, their full 3D treatment suffers severely from low spatial resolution. Because of the numerical costs, moreover until now only a few codes exist which treat at least two separate gas phases. Another serious problem is the treatment of stars. Two possibilities exist: to incorporate the stellar component into the general fluid description, or to handle a hybrid code that switches from hydrodynamics on the grid to stellar dynamics.

Although many papers denote their published numerical applications as multi-phase gasdynamics (like e.g. Wada \& Norman 1999, Wada \& Norman 2001), the simulations in reality only deal with a single gas fluid and account for a range of gas temperatures and densities. Slyz et al. 2005 studied the various effects of SF, stellar feedback, selfgravity, and spatial resolution of the evolution of the ISM structure in gaseous disks. The main conclusions which can be drawn are that without SF, the structure remains more diffuse than with SF, and that with SF stellar feedback is crucial, although at high spatial resolution the SF within grid cells inherently loses its coherence. The same is still continued in the, at present, spatially best resolved (magneto-)hydrodynamical simulations of the ISM by de Avillez \& Breitschwerdt 2004 (and more recent papers).

Nevertheless, also in such grid simulations, SF criteria within a grid cell like e.g. excess of a density threshold, excess of Jeans mass, convergence of flows, cooling timescale lower than dynamical one, fall below threshold temperature (see e.g. Tasker \& Bryan 2008) must be applied. Furthermore, at present all these applications lack the direct interactions of different gas phases by heat conduction, dynamical drag, and dynamical instabilities through forming interfaces, but the most refined ones allow to resolve the turbulence cascade.

In addition, such mixing effects at interfaces between gas phases and due to turbulence (like e.g. those in the combined SWB/HıIcomplexes) contribute to the observations by the enhancement or, respectively, dilution of metal abundances. Hydrodynamical grid models have therefore been performed that involved the elements released from nucleosynthesis in stellar progenitors of different masses and therefore also of stellar lifetimes, so-called yields. Since also the metal-dependence of astrophysical and plasmaphysical processes should be properly included in this so-called chemo-dynamics, the combined effects of gas dynamics and chemistry can be traced.

If the gasdynamics, including SF and stellar feedback, is coupled with the chemical evolution, single-gas phase simulations in $1 \mathrm{D}$ are, however, misleading. The reasons are that, at first, hot metal-enriched gas is mixed with cooler gas and, secondly, the limitations to one dimension does not allow for an escape of hot gas due to 2D shape effects. As a simple exercise, one can mix $10^{4} \mathrm{~K}$ warm gas with the ejecta of SNII explosions according to a SF efficiency of a few percent, which means that less than $1 \%$ hot gas is 
contributed to the specific energy and momentum of the warm gas phase. This results in two effects: the gas temperature does not exceed $10^{5} \mathrm{~K}$ and thus drops almost instantaneously to $10^{4} \mathrm{~K}$ again, i.e. the gas remains in its warm state; the gas dynamics of the warm gas dominates until the hot gas contributes a significant fraction to the total energy density, and the gas is already diluted by gas consumption so that the cooling is extended while the gas pressure drives an expansion. Models of that kind (Pipino, D'Ercole, \& Matteucci 2008) should be considered with caution unless two independent gas phases are treated and/or this at least in $2 \mathrm{D}$.

Since energetic effects affect the evolution of low-mass galaxies more efficiently than massive galaxies, their application to DGs is more spectacular and has been perfomed with a single gas-phase representation of the ISM, but in 2D (e.g. by Recchi \& Hensler 2006b) in order to reproduce the peculiar element abundances in Blue Compact DGs (Recchi et al. 2006b). Since gas infall not only affects the chemistry but also SF (Hensler et al. 2004b) and dynamics of outflows, we have extended the former models by infalling clouds (Recchi \& Hensler 2007) which are introduced non-self-consistently in the numerical grid as density enhancements. In these models, two kinds of cloud contributions are considered, only initially existing and continuously formed, respectively.

The issues are the following: due to dynamical processes and thermal evaporation, the clouds survive only a few tens of Myr. The internal energy of cloudy models is typically reduced by $20-40 \%$ compared to models with a smooth density distribution. The clouds delay the development of large-scale outflows, helping therefore to retain a larger amount of gas inside the galaxy. However, especially in models with continuous creation of infalling clouds, their ram pressure pierce the expanding supershells so that, through these holes, freshly produced metals can more easily escape into the galactic wind. Moreover, assuming a pristine chemical composition for the clouds, their interaction with the superbubble dilutes the hot gas, reducing its metal content. The resulting final metallicity is therefore, in general, smaller (by $\sim 0.2-0.4$ dex) than attained by smooth models.

Starting from simple 2D HD models with metallicity inclusion (Burkert \& Hensler 1987, 1988) we developed a two-gas phase chemo-dynamical prescription, which was at first applied as 1D version to the vertical formation of the Galactic disk (Burkert, Truran, \& Hensler 1992). The metallicity was treated as a general Z without differentiation of particular elements between their stellar progenitors, but with various production timescales. The model could successfully reproduce the vertical stellar density and metallicity distribution while also yielding formation timescales of the thin and thick disk components.

This chemo-dynamical scheme was further extended by us to $2 \mathrm{D}$ and to specific elements characteristic for different stellar mass progenitors and production lifetimes. This chemo-dynamical treatment includes metal-dependent stellar yields and winds, SNeIa, SNeII, PNe, metal-dependent cooling functions and heat conduction. The gas phases are described by the Eulerian hydrodynamical equations, co-existing in every grid cell in pressure equilibrium. Thereby the cool component, as representative of interstellar clouds, is described by anisotropic velocity components derived from the Boltzmann moment equations with source and sink terms from cloud collisions. The SF is parametrized as explored in Köppen, Theis, \& Hensler (1995) to guarantee self-regulation.

The application of the chemo-dynamical code to the evolution of disk galaxies (Samland \& Hensler 1996) could successfully show for the Milky Way (Samland, Hensler \& Theis 1997) how and on which timescale the components halo, bulge, and disk formed and how the abundances developed, could represent the temporal evolution of gas abundances, of the stellar radial abundance gradients, of the abundance ratios, and of star-to-gas ratios. 
This also showed how many metals are stored in the hot gas phase and mixed with the cool one by evaporation of clouds and condensation.

Until now, the optimal grid code is considered to be the further development of the chemo-dynamical scheme (Samland, Hensler \& Theis 1997) to 3D and with stellar dynamics for the stars. In addition, a cosmologically growing DM halo is included in the simulations by Samland \& Gerhard (2003). These models contain all the crucial processes of SF self-regulation by stellar feedback, multi-phase ISM, and temporally resolved stellar components according to the chemo-dynamical prescription (Hensler 2003, 2007). They cannot only trace the formation and evolution of the disk galaxies' components, but also of characteristic chemical abundances, and are until now the best self-consistent evolutionary models of disk galaxies, because the disk formation is included into the global temporal galaxy evolution. Moreover, the formation and evolution of disk galaxies in their infancy were studied by these self-consistent simulations with respect to bulge formation and disk fragmentation (Immeli et al. 2004).

\section{Discussion}

While SPH codes appear plausibly to be the most appropriate numerical treatment of galaxy evolution including gaseous disks, it must be considered with caution that for the multi-component representation with interaction processes the particle number is still at present limited to a few 10,000, which means that gas packages and stellar particles in massive galaxies represent unrealistically large masses. Only for DGs does this effort lead to acceptable resolutions.

I am here not advocating against particle codes, and I am optimistic that advances in computer facilities and numerical codes will soon allow to overcome the spatial resolution problem and allow for multi-component descriptions. Nevertheless, at the moment grid strategies look much more promising because small-scale processes are more easily and reliably coupled with large-scale dynamics. In addition, physical processes can be treated more appropriately. This also allows an outlook if the self-consistent global models are further developed to include magnetic fields, radiative and Cosmic-ray transport.

A word of care should be expressed with respect to empirical relations. Agreement with the Kennicutt relation is achieved for simulations which are totally differing in the treatment of feedback processes, while the $\mathrm{H} \alpha$ surface brightness relation deviate from the Kennicutt slope of 1.4 with atomic Hi gas, molecular gas, and for different modes of SF, so any representation of this relation by any modelling of galaxy evolution seems to be too weak to validate any numerical treatment by itself. Likewise, metallicity gradients and age-metallicity relations in galaxy disks also seem not to be sufficient for the validation of numerical codes. This means, on the other hand, that issues of such relations and the requirement to disentangle inherent evolutionary processes are overestimated, and that these relations are robust and insensitive to the intrinsic state of galaxies.

Furthermore, the presented models show that it seems illusory to try to correlate SF with gas densities if feedback processes are neglected.

We are still far from fully self-consistent models that allow for the inclusion of the necessary astrophysical and plasmaphysical processes on all length and time scales required. Growing computer facilities will allow soon for 3D AMR simulations, but not only refined spatial and temporal resolution is the clue; also coherence on different scales as necessary for SF, turbulence, magnetic fields, etc. require intuition, brain waves, and a large effort of hard code developing and testing. 


\section{Acknowledgements}

The author is gratefully acknowledging collaborations and discussions on this topic with Peter Berczik, Dieter Breitschwerdt, Stefan Harfst, Stefan Hirche, Joachim Köppen, Simone Recchi, Andreas Rieschick, Markus Samland, Rainer Spurzem, Christian Theis, Wolfgang Vieser, and Hervé Wozniak. I also thank Simone Recchi for his careful reading of the manuscript and the organizers cordially for their invitation to this conference and their support. This work is supported by the key programme "Computational Sciences" of the University of Vienna under project no. FS538001.

\section{References}

Barnes, J. \& Hut, P. Nature, 324, 446

Benjamin, R. \& Danly, L. 1997, ApJ, 481, 764

Berczik P., 1999, A\&A, 348, 371

Berczik, P., Hensler, G., Theis, C., \& Spurzem, R. 2003, Ap\&SS, 284, 865

Berczik, P., Hensler, G., Theis, C., et al. 2009, A\&A, submitted

Böhringer, H. \& Hensler, G. 1989, A\&A, 215, 147

Bouche, N., Cresci, G., Davies, R., et al. 2007, ApJ, 671, 303

Bournaud, F., Elemegreen, B. G., \& Elmegreen, D.M. 2007, ApJ, 670, 237

Burkert, A. \& Hensler, G. 1987, MNRAS, 225, 21p

Burkert, A. \& Hensler, G. 1988, A\& A, 199, 131

Burkert, A., Truran, J., \& Hensler, G. 1992, ApJ, 391, 651

Cox, D.P., 2005, ARAA, 43, 337

de Avillez, M. A. \& Breitschwerdt, D. 2004, A\&A, 425, 899

Dalla Vecchia, C. \& Schaye, J. 2008, MNRAS, 387, 1431

Ehlerova, S., Palous, J., Theis, C., \& Hensler, G. 1997, A\&A, 328, 111 1997, ApJ, 481

Elmegreen, B.G. 2002, ApJ, 577, 206

Elmegreen, B. G. \& Efremov, Y. N. 1997, ApJ, 480, 235

Ferrière, K. M., 2001, Rev. Mod. Phys., 73, 1031

Fraternali, P., van Moorsel, G., Sancisi, R., \& Oosterloo, T. AJ, 123, 3124

Freyer, T., Hensler, G., \& Yorke, H. W., 2003, ApJ, 594, 888

Freyer, T., Hensler, G., \& Yorke, H. W., 2006, ApJ, 638, 262

Fukuda, N. \& Hanawa, T. 2000, ApJ, 533, 911

Gao, Y. \& Solomon, P. M., 2004, A\&A, 606, 271

Harfst, S., Theis, C., \& Hensler, G. 2006, A\&A, 499, 509

Hensler, G. 2003, ASP Conf. Ser. Vol., 304, eds. C. Charbonnel et al., p. 371

Hensler, G. 2007, EAS Publ. Ser. Vol., 24, eds. E. Ensellem et al., p. 113

Hensler, G., Dickow, R., Junkes, N., \& Gallagher, J.S., III. 1998, ApJ, 502, L17

Hensler, G., Kppen, J., Pflamm, J., \& Rieschick, A. 2004, IAU Symp. Ser. 217, eds. P.-A. Duc, J. Braine, E. Brinks, p. 178

Hensler, G. \& Rieschick, A. 2002, ASP-CS, 285, 341

Hensler, G., Theis, Ch., \& Gallagher, J. S., III. 2004, A\&A, 426, 25

Heyer, M. H., Brunt, C., Snell, R. L., et al. 1996, ApJ, 464, L175

Heyer, M. H., Corbell, E., Schneider, S. E., \& Young, J.S., 2004, ApJ, 602, 723

Immeli, A., Samland, M., Gerhard, O., \& Westera, P. 2004, A\&A, 413, 547

Kennicutt, R. J. 1998, ApJ, 498, 541

Keres, D., Katz, N., Weiberg, D. H., \& Dave, 2005, MNRAS, 363, 2

Knauth, D. C., Meyer, D. M., \& Lauroesch, J. T. 2006, ApJ, 647, L115

Köppen, J. \& Hensler, G. 2005, A\& A, 434, 531

Köppen, J., Theis, C., \& Hensler, G. 1995, A\&A, 296, 99

Köppen, J., Theis, C., \& Hensler, G. 1998, A\&A, 328, 121

Krumholz, M. R. \& McKee, C. F. 2005 ApJ, 630, 250 
Kravtsov, A. V. 2003, ApJ, 590, L1

Larson, R. B. 1969, MNRAS, 145, 405

Larson, R. B. 1974, MNRAS, 169, 229

Larson, R. B. 1975, MNRAS, 173, 671

Larson, R. B. 1976, MNRAS, 176, 31

Lasker, B. M. 1967, ApJ, 149, 23

Li, Y., MacLow, M.-M., \& Klessen, R. S. 2005, ApJ, 626, 823

MacLow, M.-M. \& Klessen, R. S. 2004, Rev.Mod.Phys., 76, 125

Martin, C. L., Kobulnicki, H. A., \& Heckman, T. M. 2002, ApJ, 574, 663

Mühle, S., Klein, U.,Wilcots, E. M., \& Hüttermeister, S. 2005, AJ, 130, 524

Ostriker, E., Stone, J. M., \& Gammie, C. F. 2001, ApJ, 546, 980

Pipino, A., DErcole, A., \& Matteucci, F. 2008, A\&A, 484, 679

Rana, N. C. \& Wilkinson, D. A. 1986, MNRAS, 218, 497

Recchi, S. \& Hensler, G. 2006a, A\&A, 445, L39

Recchi, S. \& Hensler, G. 2006b, Rev. Mod. Astronomy, 18, 164

Recchi, S. \& Hensler, G. 2007, A\& A, 476, 841

Recchi, S., Hensler, G., Angeretti, L., \& Matteucci, F. 2006, A\&A, 445, 875

Rieschick, A. \& Hensler, G. 2000, ASP-CS, 215, 130

Samland, M. \& Hensler, G. 1996, Rev. Mod. Astron., 9, 277

Samland, M., Hensler, G., \& Theis, C. 1997, ApJ, 476, 544

Samland, M. \& Gerhard, O. 2003, A\&A, 399, 961

Sancisi, R., Fraternali, F., Oosterloo, T., \& van der Hulst, T. 2008, A\&A Rev, 15, 189

Scannapieco, C., Tissera, P. B., White, S. D. M, \& Springel, V. 2006, MNRAS, 371, 1125

Schaye, J. \& Dalla Vecchia, C. 2008, MNRAS, 383, 1210

Schmidt, M. 1959, ApJ, 129, 243

Semelin, B. \& Combes, F. 2002, A\&A, 388, 826

Slyz, A. D., Devriendt, J. E. G., Bryan, G., \& Silk, J. 2005, MNRAS, 356, 737

Springel, V. 2000, MNRAS, 312, 859

Springel, V., Yoshida, N., \& White, S. D. M. 2001, New Astron., 6, 79

Stil, J. M. \& Israel, F. P. 2002, A\&A, 392, 473

Strickland, D. K., Heckman, T. M., Colbert, E. J. M., et al. 2004, ApJ, 606, 829

Tasker, E. J. \& Bryan, G. L. 2008, ApJ, 673, 810

Thornton, K., Gaudlitz, M., Janka, H.-Th., \& Steinmetz, M. 1998, ApJ, 500, 95

Vieser, W. \& Hensler, G. 2007, A\&A, 472, 141

Wada, K. \& Norman, C. A. 1999, ApJ, 516, L13

Wada, K. \& Norman, C. A. 2001, ApJ, 547, 172

Wada, K. \& Norman, C. A. 2007, ApJ, 660, 276

Wise, J. H. \& Abel, T. 2007, ApJ, 665, 899

Wolfire, M. G., McKee, C. F., Hollenbach, D., \& Tielens, A. G. G. M. 1995, ApJ, 453, 673 